\title{
Learning from myocarditis: mimicry, chaos and black holes
}

\author{
Noel R. Rose
}

Address: Departments of Pathology and of Molecular Microbiology and Immunology, The Johns Hopkins Schools of Medicine and Public Health, 615 North Wolfe Street, Baltimore, MD 21205, USA

Email: nrrose@jhmi.edu

Fl000Prime Reports 2014, 6:25 (doi:10.12703/P6-25)

All FI000Prime Reports articles are distributed under the terms of the Creative Commons Attribution-Non Commercial License (http://creativecommons.org/licenses/by-nc/3.0/legalcode), which permits non-commercial use, distribution, and reproduction in any medium, provided the original work is properly cited.

The electronic version of this article is the complete one and can be found at: http://fl000.com/prime/reports/b/6/25

\begin{abstract}
Autoimmune myocarditis and its sequel, dilated cardiomyopathy, are major causes of heart failure, especially in children and young adults. We have developed animal models to investigate their pathogenesis by infecting genetically susceptible mice with coxsackievirus B3 or by immunizing them with cardiac myosin or its immunodominant peptide. A number of valuable lessons have emerged from our study of this paradigm of an infection-induced autoimmune disease. We understand more clearly how natural autoimmunity, as an important component of normal physiology, must be recalibrated regularly due to changes caused by infection or other internal and external stimuli. A new normal homeostatic platform will be established based on its evolutionary fitness. A loss of homeostasis with out-of-control normal autoimmunity leads to autoimmune disease. It is signified early on by a spread of an adaptive autoimmune response to novel epitopes and neighboring antigens. The progression from infection to normal, well-balanced autoimmunity to autoimmune disease and on to irreversible damage is a complex, step-wise process. Yet, chaos theory provides hope that the pattern is potentially predictable. Infection-induced autoimmune disease represents a sequence of events heading for a train wreck at the end of the line. Our aim in autoimmune disease research must be to stop the train before this happens.
\end{abstract}

\section{Myocarditis and dilated cardiomyopathy}

Defined as mononuclear inflammation of the heart muscle with damage to adjacent myocytes, myocarditis is a major cause of sudden death in adolescents and young adults [1-3]. In about $21 \%$ of cases, myocarditis evolves into chronic inflammatory dilated cardiomyopathy, which further progresses to heart failure $[4,5]$. The outcome of dilated cardiomyopathy is poor with a 5 year mortality of $46 \%$ [1]. Although in the majority of cases the cause of myocarditis remains unknown, a great deal of evidence supports an association with a prior viral infection. The most frequently associated viruses include coxsackievirus B3, adenovirus, parvovirus B19, cytomegalovirus, Epstein-Barr virus, human immunodeficiency virus and hepatitis viruses A and C.

Coxsackievirus $\mathrm{B} 3$, an enterovirus in the picornavirus family, is a non-enveloped positive-stranded RNA virus [6]. Commonly associated with mild upper respiratory or gastrointestinal symptoms, infected individuals may develop acute viral myocarditis. It is attributed to a direct cytopathic effect of coxsackievirus B3 on cardiomyocytes. The great majority of patients recover spontaneously from acute viral myocarditis, but approximately $10 \%$ of cases evolve into chronic myocarditis [7]. Although viral RNA can often be identified in the cardiac tissue of such myocarditis patients, an intact infectious virus is not evident [8]. At the same time, many of the chronic myocarditic patients develop heart-reactive autoantibodies, suggesting an autoimmune mechanism $[1,9,10]$. Cardiac antibodies include those specific for intracellular antigens, including cardiac myosin heavy chain, cardiac troponin I, mitochondrial antigens (such as adenine nucleotide translocator) and branched-chain alpha-keto dehydrogenase [2]. In addition, antibodies to cardiac cell surface receptors, especially the $\beta 1$-adrenergic receptor and the M2 muscarinic receptor, have been described. It is still not known how many of these antibodies play a 
role in the pathogenesis of chronic myocarditis and dilated cardiomyopathy.

There are several distinct histologic and clinical forms of chronic myocarditis [1]. In lymphocytic myocarditis, the extensive infiltration of monocytes and lymphocytes is seen in the myocardium with evidence of myocyte necrosis. This form of the disease can usually be managed successfully, especially if diagnosed early [3]. Giant cell myocarditis and necrotizing eosinophilic myocarditis (less common forms of the disease) have especially high fatality rates. Establishing a definitive diagnosis of chronic myocarditis usually requires an endomyocardial biopsy.

Among cases of chronic lymphocyte myocarditis that fail to resolve spontaneously, a subpopulation proceeds to dilated cardiomyopathy [1]. This disease is characterized by left ventricular dilatation and impaired cardiac output. Dilated cardiomyopathy patients are prone to congestive heart failure with a 5 year mortality rate of $46 \%$. In the cases where supportive cardiac treatment fails, survival requires cardiac transplantation. Histologically, dilated cardiomyopathy is characterized by extensive replacement of heart muscle cells with fibroblasts and deposition of collagen [5]. Described as cardiac remodeling, it represents extensive healing. A fibrotic heart is not functional, so that dilated cardiomyopathy represents the end of a long train of events initiated by viral infection, progressing to an inflammatory autoimmune disease and concluding in an irreversible fibrotic outcome - the wreck at the end of the train ride [6].

\section{Constructing a model}

The evidence cited above suggests autoimmunity as a plausible mechanism in driving the train of events from a virus infection to autoimmune inflammation. Since coxsackievirus B3 was the virus most often implicated in human myocarditis, we selected this agent to devise an experimental model suitable for detailed mechanistic studies. Fortunately, well-studied models of coxsackievirus B3-induced myocarditis in mice were available from the investigations of Sally Huber and her colleagues $[11,12]$. Since coxsackievirus B3 infection is quite common, but chronic myocarditis is relatively uncommon, we started with the premise that an immunopathic outcome depends upon restricted genetic susceptibility. For that reason, we infected some 25 genetically different strains of inbred mice with a well-studied cardiotropic strain of coxsackievirus B3 Nancy strain virus [13]. All strains of mice tested developed acute myocarditis starting 2 or 3 days after coxsackievirus B3 infection. Inflammation reached its peak about day 7 and gradually resolved in most mice so that by day 21, the heart appeared histologically normal. Viral genome persisted, but we detected no infectious virus in the heart after day nine. In a few strains of mice, myocarditis persisted, but with a distinct change in the histologic picture. Furthermore, the mouse strains that developed this chronic phase of myocarditis produced autoantibodies specific for cardiac myosin heavy chain, resembling those we had seen in patients with chronic myocarditis.

These findings provided the basis for our premise that chronic myocarditis represents a heart-specific autoimmune response initiated by the viral infection. In order to support this hypothesis, we immunized mice with purified mouse cardiac myosin [14]. All of the mouse strains susceptible to chronic myocarditis following coxsackievirus B3 infection developed disease approximating post-viral myocarditis. In contrast, C57/BL10 and C57/BL6 mice, which are resistant to post-infection chronic myocarditis, failed to develop disease following immunization with purified mouse cardiac myosin. No heart lesions were found in any of the controls that received purified skeletal myosin rather than cardiac myosin, in accordance with the organ-specific character of the disease.

To provide further evidence that this inflammatory autoimmune heart disease is due to the response to cardiac-specific myosin, experiments were done tolerizing genetically susceptible mice to cardiac myosin $[15,16]$. Genetically susceptible mice tolerized to cardiac myosin failed to develop chronic myocarditis following viral infection.

This finding initially suggested that the chronic phase of myocarditis represents an immune response intiated by T cell mimicry between an epitope on the coxsackievirus B3 virus and cardiac myosin. Extensive evidence supporting this view was reviewed by Madeleine Cunningham [17]. On the other hand, Gauntt et al. [18] and Horwitz et al. [19] produced evidence showing that the autoimmune myocarditis involves virus inflicting damage to the heart. These findings suggest the alternative interpretation that virus infection injures the myocytes. The damage mobilizes intracellular cardiac myosin and releases the alarmins required for the adjuvant effect. In our hands, the immunization of mice with concentrated inactivated coxsackievirus B3 with complete Freund's adjuvant consistently failed to induce cardiac-specific antibodies or myocarditis even in highly susceptible A/J strains of mice $[13,20]$.

To establish definitively that chronic myocarditis is caused by an autoimmune response, adoptive transfer experiments were conducted using donors immunized with either cardiac myosin or a purified peptide of 
cardiac myosin. The transfer of myosin-specific CD4 $\mathrm{T}$ cells induced autoimmune myocarditis in appropriately prepared syngeneic recipients [21]. The development of myocarditis was fully inhibited by a monoclonal antibody to CD4 helper T cells and genetic susceptibility resides in the CD4 T cell [22]. The contribution of the CD8 T cell is still unsettled because it can either suppress or promote myocarditis depending on the time and method of depletion $[23,24]$.

The finding that susceptibility to autoimmune myocarditis is genetically restricted provided a unique opportunity to delineate genetic control of an infection-induced autoimmune disease [25]. H2 haplotype affects the response since A/J and A.SW mice develop severe disease, whereas A.BY mice (with the same background genes) produce minimal lesions. Unlike most autoimmune diseases, further study showed that non-H2 background genes are especially prominent in determining susceptibility. For example, A.SW mice developed severe autoimmune myocarditis whereas B10.S mice, sharing the same $\mathrm{H} 2$ genes, show only limited response to immunization with purified cardiac myosin. Genome-wide linkage analysis revealed at least 2 loci had significant effects on the susceptibility to autoimmune myocarditis: eam-1 located on the proximal end of chromosome 1 and eam-2, which is distal on chromosome 6 [26]. Both of these chromosomal segments bear genes determining susceptibility to a number of other autoimmune diseases. We proposed that multiple genes controlling normal immunoregulatory processes are key in determining autoimmunity, with chance aggregation of particular alleles contributing to greater susceptibility to autoimmune disease.

Although most autoimmune diseases are more common in women, myocarditis in humans is an example of an autoimmune disease with greater prevalence in men. Similarly, in the experimental models, either induced by viral infection or by immunization with cardiac myosin, male mice are more prone to develop severe disease [27]. Frisancho-Kiss et al. showed that the increased inflammation in male mice is not due to greater viral replication in the heart, but rather associated with increased cytokines, interleukin (IL)-1 $\beta$, IL-1-18 and interferon (IFN)- $\gamma$ [28]. To study the effect of male sex hormones on the cardiac inflammation, Frisancho-Kiss gonadectomized male BALB/c mice prior to infection with coxsackievirus B3 and found a shift in the macrophage/ monocyte population with a greater percentage of M2 macrophages in the gonadectomized males [29]. Similar findings have been described using the cardiac myosininduced disease [30]. These findings help to align the experimental models with the human disease.
In addition to identifying genes determining susceptibility to autoimmune myocarditis, the experimental models lend themselves in functional studies to trace the critical steps leading to autoimmune myocarditis. These studies have recently been summarized [31]. For example, two early cytokines, IL-1 $1 \beta$ and tumor necrosis factor- $\alpha$ are necessary and sufficient for determining the progression from initial viral myocarditis to later autoimmune myocarditis. Additional evidence showing that the early innate immune response to the virus dictated subsequent autoimmune myocarditis came from experiments administering bacterial lipopolysaccharide at the time of viral infection. Activation of innate immunity through toll-like receptor (TLR) 3 induced later autoimmune myocarditis even in genetically resistant C57BL/ 6 mice. Although a single cytokine does not predict oncoming autoimmune disease, we found that patterns of inflammatory cells and their cytokine products predicted spontaneous resolution, autoimmune lymphocytic myocarditis, dilated cardiomyopathy or eosinophilic myocarditis following viral infection.

\section{Placing the blame}

The studies described above identify the $\mathrm{CD} 4+\mathrm{T}$ cell as an orchestrator of the autoimmune response in myocarditis. The presence of cardiac myosin-specific $T$ cells in the periphery of normal mice is evidence that $\mathrm{T}$ cells with this specificity are not fully deleted during their education in the thymus. Normally, self-reactive T cells are continually well regulated and controlled. Sometimes these few naturally occurring self-reactive $\mathrm{T}$ cells can be strongly stimulated and lead to autoimmune disease. Virus infection itself is a potent $\mathrm{T}$ cell stimulator, as is its surrogate, complete Freund adjuvant. Coxsackieviruses are ideally qualified to prime $\mathrm{T}$ cells because they can provide both the antigen-specific signals by injuring heart muscle cells and antigen-non-specific adjuvant signals used for $\mathrm{T}$ cell activation [32].

Both the quantity and quality of activation signals given to $\mathrm{T}$ cells are shaped by antigen-presenting cells such as the dendritic cell. Non-antigen-specific signals act as an adjuvant and determine the balance between up- and down-regulatory effects [33]. The strength of the antigenspecific signal depends upon the effectiveness of clonal deletion. The affinity of $\mathrm{T}$ cell receptor binding and the availability of the antigen are all factored into the final strength of the antigen-specific signal. Once adaptive immunity inaugurates $\mathrm{T}$ cell proliferation, the initially targeted epitope-specific response begins to amplify. In the case of an autoimmune response, the broadening may involve both intramolecular spread to additional epitopes on the myosin antigen or, even more striking, extension to additional cardiomyocyte antigens. For 
example, immunization with troponin I later gives rise to antibodies to cardiac myosin [10]. Clinically, the appearance of autoantibodies directed to multiple cardiac-specific antigens is indicative of a mounting autoimmune response.

Several varied effector pathways dictate the course of disease progression and its outcome. In the case of autoimmune myocarditis, all three of the major helper $\mathrm{T}$ cell effector pathways are engaged in producing pathogenic autoimmunity [34]. Different forms of myocarditis are associated with earlier or greater production of IL-12 (the Th1 inducer), IL-4 (a T helper cell type 2 [Th2] inducer) and IL-23 (required for continued Th17 response). The subsequent immune response does not always follow the prototypic pathways. IFN- $\gamma$, the customary signature cytokine of a Th1 response, retards the development of myocarditis, perhaps by suppressing the Th1 response and favoring Th17. On the other hand, IL-13 (a cytokine associated with Th2 responses) diminishes disease, in contrast to IL-4 which enhances disease [31].

The changing cytokine profile dictates dramatic differences in disease outcome. The administration of anti-IL-4 antibody or genetic knockout greatly decreases the severity of the eosinophilic and giant cell myocarditis characteristic of $\mathrm{A} / \mathrm{J}$ mice [34]. IL-17, a cytokine long associated with neutrophilic inflammation, has little impact on the overall severity of inflammation in autoimmune myocarditis at its peak on day 21 in BALB/C mice but is critical for later cardiac remodeling and dilated cardiomyopathy [35]. Unexpectedly, IL-17 acts on cardiac fibroblasts to promote a fibrotic response. The balance of mediators acts to tailor the inflammatory response. As stated previously, the IFN- $\gamma$-deprived mice developed a particularly severe inflammatory myocarditis. Crossing an IFN- $\gamma$ deficient animal with an IL-17A deficient mouse produces a strain that, on immunization with cardiac myosin, produces a rapidly fatal eosinophilic myocarditis [36]. It resembles the life-threatening necrotic eosinophilic myocarditis seen in humans. These experiments provide hints that the precise profile of inflammatory cells and their cytokine products as they evolve early during the course of disease predicts the eventual disease outcome.

\section{Clonal deletion and molecular mimicry - two sides of the coin}

In explaining infection-induced autoimmunity, molecular (or, more precisely, epitope) mimicry is the most cited mechanism [37]. Indeed, there are several wellestablished examples where a particular antigen or combination of antigens from a microorganism are actually able to reproduce the cardinal features of a relevant autoimmune disease under experimental conditions. The $\beta$-hemolytic streptococcus, a bacterium clinically and epidemiologically associated with rheumatic fever, has been shown in a mouse or rat to elicit both the myocarditis and endocarditis characteristic of rheumatic heart disease. Similarly, antigens from Campylobacter jejuni, a common bacterial inhabitant of the intestine, on experimental immunization produce evidence of neurologic dysfunction suggestive of Guillain-Barre syndrome.

Beyond these examples of actual induction of disease, a vast number of instances of epitope mimicry between microorganisms and human cellular antigens have been described. In the great majority of instances, there is still no evidence, however, that the antigenic mimic is capable of inducing an autoimmune disease.

The preponderance of evidence indicates that the clonal deletion of self-reactive $\mathrm{T}$ cells is rarely complete. Elimination of T cells in the thymus depends upon the affinity of their receptor with antigen-presenting medullary cells [38]. Negative selection ranges from complete clonal deletion to none. Complete deletion takes place with only a few major antigens, such as the dominant carbohydrate moieties of the major $\mathrm{ABO}$ blood group antigens [39]. Autoantibodies are never produced to these antigens by subjects who present the respective antigen. At the other end of the spectrum are strictly tissue-limited organ-specific antigens such as the lens and the sperm. It is relatively easy to induce autoantibodies to these antigens. Thymic presentation of selfantigens is essential for normal self-tolerance. Some organ-specific antigens are mainly presented in the thymus through the agency of autoimmune regulatory (AIRE) genes. Mice lacking these genes spontaneously develop different organ-specific diseases, their form depending primarily on the MHC haplotype of the mouse. Most other self-antigens are somewhere on this continuum from inducing no autoimmunity to producing pronounced autoimmune responses.

The fact that clonal deletion is generally incomplete means that there is an ever-present risk of loci of self tolerance and developing autoimmune disease. Yet, the long-range danger that would arise from complete deletion of all self-reactive $\mathrm{T}$ cells is even greater. The extensive sharing of epitopes between microorganisms and the human body might entail complete removal of all cross-reacting and self-reactive $\mathrm{T}$ cell precursors. It would narrow the ability of the human host to recognize and respond to many of the critical epitopes of invading pathogenic microorganisms. Inevitably, it 
would lead to major deficits in the host's protective immunity. Such infamous "black holes" in the protective immune repertoire, rendering the host susceptible to infectious disease, would be strongly selected against by evolution. In short, despite molecular mimicry and its risk of inducing autoimmune disease, maintaining a full repertoire of $\mathrm{T}$ lymphocytes is required to recognize present and future pathogenic microorganisms.

The argument on the B cell side is even clearer. Selfreactive "natural" antibodies are well represented in the normal circulation. Although usually of low affinity and highly cross-reactive, natural autoantibodies represent an important first-line defense in the innate immune system. They are often dismissed as "background noise". The longstanding practice in clinical immunology laboratories has been to dilute serum samples several fold before testing for disease-associated autoantibodies to avoid "false positive" tests. The persistence of mature autoantibodies over time suggests that they represent an important survival mechanism in an environment filled with potential pathogens.

Since most self-reactive $\mathrm{T}$ cells are not deleted during development, various mechanisms have evolved to control them [40]. At this time, the best studied candidates for a regulatory mechanism depend upon a specialized population of regulatory $\mathrm{CD} 4 \mathrm{~T}$ cells. The history of these cells goes back to early studies on neonatal or post-irradiation thymectomy, which can lead to the spontaneous occurrence of organ-specific autoimmune disease [41]. The important function of these cells is well demonstrated in mice, but therapeutic applications in humans have been less clear cut. This suggests that classical regulatory $\mathrm{T}$ cells represent just one of a large number of agents for maintaining selftolerance. Increasing evidence points to $\mathrm{B}$ cells and myeloid cells as additional directors of peripheral selftolerance because they are in an ideal position to influence antigen-specific and non-antigen-specific unresponsiveness. As examples of normal immune regulators, cytotoxic T-lymphocyte antigen, PD-1 and PD-L-1 have been identified, and their involvement in preventing myocarditis and other autoimmune diseases has been reported. It is likely that the sum total of multiple, subtle regulatory factors, specific and non-specific, makes the pivotal difference between everyday harmless natural autoimmunity and a pathogenic autoimmune train wreck.

\section{Chaos and prediction}

The goal of the immune system, as with all of the physiologic systems of the body, is to maintain the stable platform from which the essential functions of life can be performed and sustained [42]. Functionally the most successful homeostatic state has been selected over time by evolution. Evolution related to immunology is ever changing. Macroevolution over millennia has provided humans with both an innate and an adaptive immune system, and selected mechanisms for optimal protection against pathogenic microorganisms. Microevolution is represented by the changes constantly occurring over the life span. They include the regular alterations in form and function within the body from birth to death. External influences include physical, chemical and infectious agents encountered in the environment. The microbiologic inhabitants of the body (the microbiome) live in a carefully balanced state of mutual accommodation and interact intimately with the immune system. The normal immune system responds to a constantly changing spectrum of challenges to the survival and reproduction of the host.

Because the elements that go into maintaining immunologic homeostasis are incredibly complex and intimately interactive, a change in any one of the myriad components is reflected throughout the system. Identifying and understanding changes in a simple component of the system separately at any point of time would not enable us to predict the final outcome. The immune system represents a classical example of chaos.

Several decades ago, mathematicians developed tools for looking at natural systems, such as weather. While starting with the assumption that the only prediction one can make about a complex system is that it is unpredictable, chaos theory discerned certain patterns that are repeatable and can be applied to similar situations. Although every snowflake is different, the overall six-branched structure is absolutely predictable.

Our studies of myocarditis over the years convinced us that we can recognize overall patterns in the course of infection and the subsequent development of autoimmunity that will allow us to predict the eventual outcome. Our goal remains to determine, at the earliest stages, when an autoimmune response has exceeded the boundaries of normal homeostatic immunoregulation and is progressing from natural autoimmunity to autoimmune disease. Intervening before irreversible damage has occurred should enable us to arrest or even reverse autoimmune disease. We seek those tell-tale patterns in the prior infectious process that warn of a pathogenic deviation from immunologic homeostasis. In experimental models, we have discovered that distinctive cytokine patterns during early inflammation tell us what we can expect: a rapidly evolving eosinophilic myocarditis, a slow progression to dilated cardiomyopathy or remission and recovery. 


\section{Abbreviations}

IFN- $\gamma$, Interferon gamma; IL, Interleukin; Th2, T helper cell type 2.

\section{Disclosures}

The author declares that he has no disclosures.

\section{Acknowledgements}

Our research work was supported by NIH grants HL077611, HL067290 and HL113008. I am pleased to thank Ms. Starlene Murray for her editorial assistance.

\section{References}

I. Rose NR, Kaya Z: From Myocarditis and dilated cardiomyopathy. In The Autoimmune Diseases. 5th edition. Edited by Rose NR, Mackay I. San Diego: Elsevier; 2014:I033-44.

2. Canter CE, Simpson KP: Diagnosis and treatment of myocarditis in children in the current era. Circulation 2014, I29:1 I5-28.

3. Eckart RE, Shry EA, Burke AP, McNear JA, Appel DA, CastilloRojas LM, Avedissian L, Pearse LA, Potter RN, Tremaine L, Gentlesk PJ, Huffer L, Reich SS, Stevenson WG: Sudden death in young adults: an autopsy-based series of a population undergoing active surveillance. J Am Coll Cardiol 20I I, 58:I254-6I.

\section{FlOOOPrime}

4. Kawai C: From myocarditis to cardiomyopathy: mechanisms of inflammation and cell death: learning from the past for the future. Circulation 1999, 99:1091-100.

\section{FlOOOPrime \\ RECOMMENDED}

5. Kodama M, Hanawa H, Saeki M, Hosono H, Inomata T, Suzuki K, Shibata A: Rat dilated cardiomyopathy after autoimmune giant cell myocarditis. Circ Res 1994, 75:278-84.

6. Afanasyeva M, Rose NR: From Viral Infection and Heart Disease: Autoimmune Mechanisms. In: Infection and Autoimmunity. Edited by Shoenfeld Y, Rose NR. San Diego: Elsevier; 2004:299-318.

7. Anzini M, Merlo M, Sabbadini G, Barbati G, Finocchiaro G, Pinamonti B, Salvi A, Perkan A, Di Lenarda A, Bussani R, Bartunek J, Sinagra $G$ : Long-term evolution and prognostic stratification of biopsy-proven active myocarditis. Circulation 2013, I 28:2384-94.

\section{FIOOOPrime}

8. Chapman NM, Kim KS: Persistent coxsackievirus infection: enterovirus persistence in chronic myocarditis and dilated cardiomyopathy. Curr Top Microbiol Immunol 2008, 323:275-92.

9. Caforio AL, Mahon NJ, Mckenna WJ: Cardiac autoantibodies to myosin and other heart-specific autoantigens in myocarditis and dilated cardiomyopathy. Autoimmunity 200I, 34:199-204.

\section{FlOOOPrime}

10. Kaya Z, Katus HA, Rose NR: Cardiac troponins and autoimmunity: their role in the pathogenesis of myocarditis and of heart failure. Clin Immunol 2010, I34:80-8.

II. Liu W, Dienz O, Roberts B, Moussawi M, Rincon M, Huber SA: IL-2 I R expression on CD8+ $T$ cells promotes $C D 8+T$ cell activation in coxsackievirus B3 induced myocarditis. Exp Mol Pathol 2012, 92:327-33.

\section{FlOOOPrime}

\section{RECOMMENDED}

12. Liu W, Moussawi M, Roberts B, Boyson JE, Huber SA: Crossregulation of $\mathrm{T}$ regulatory-cell response after coxsackievirus
B3 infection by NKT and $\gamma \delta$ T cells in the mouse. Am J Pathol 2013, I 83:44I-9.

FIOOOPrime
RECOMMENDED

13. Rose NR, Beisel KW, Herskowitz A, Neu N, Wolfgram LJ, Alvarez FL, Traystman MD, Craig SW: From Cardiac Myosin and Autoimmune Myocarditis. In: Autoimmunity and Autoimmune Disease, No. 129. Ciba Foundation Symposium. Edited by Evered D, Whelan J. Chichester: Wiley \& Sons, Ltd; 1987:3-24.

14. Neu N, Rose NR, Beisel KW, Herskowitz A, Gurri-Glass G, Craig SW: Cardiac myosin induces myocarditis in genetically predisposed mice. J Immunol 1987, 139:3630-6.

15. Wang Y, Afanasyeva M, Hill SL, Kaya Z, Rose NR: Nasal administration of cardiac myosin suppresses autoimmune myocarditis in mice. J Am Coll Cardiol 2000, 36:1992-9.

16. Fousteri G, Dave A, Morin B, Omid S, Croft M, Herrath MG von: Nasal cardiac myosin peptide treatment and OX40 blockade protect mice from acute and chronic virally-induced myocarditis. J Autoimmun 201 I, 36:210-20.

\section{FlOOOPrime}

\section{RECOMMENDED}

17. Cunningham MW: T cell mimicry in inflammatory heart disease. Mol Immunol 2004, 40: I I 2 I-7.

\section{FlOOOPrime}

RECOMMENDED

18. Gauntt CJ, Trousdale MD, LaBadie DR, Paque RE, Nealon T: Properties of coxsackievirus B3 variants which are amyocarditic or myocarditic for mice. J Med Virol 1979, 3:207-20.

\section{FlOOOPrime} RECOMMENDED

19. Horwitz MS, La Cava A, Fine C, Rodriguez E, llic A, Sarvetnick N: Pancreatic expression of interferon-gamma protects mice from lethal coxsackievirus B3 infection and subsequent myocarditis. Nat Med 2000, 6:693-7.

\section{FlOOOPrime \\ RECOMMENDED}

20. Neu N, Craig SW, Rose NR, Alvarez F, Beisel KW: Coxsackievirus induced myocarditis in mice: cardiac myosin autoantibodies do not cross-react with the virus. Clin Exp Immunol 1987, 69:566-74.

21. Smith SC, Allen PM: Myosin-induced acute myocarditis is a $\mathbf{T}$ cell-mediated disease. J Immunol I99I, I47:2141-7.

\section{FIOOOPRIME
RECOMMENDED}

22. Chen P, Baldeviano GC, Ligons DL, Talor MV, Barin JG, Rose NR, Cihakova D: Susceptibility to autoimmune myocarditis is associated with intrinsic differences in CD4(+) $\mathrm{T}$ cells. Clin Exp Immunol 2012, 169:79-88.

23. Smith SC, Allen PM: The role of $\mathbf{T}$ cells in myosin-induced autoimmune myocarditis. Clin Immunol Immunopathol I993, 68: I00-6.

24. Penninger JM, Pummerer C, Liu P, Neu N, Bachmaier K: Cellular and molecular mechanisms of murine autoimmune myocarditis. APMIS 1997, I05:I-I3.

25. Li HS, Ligons DL, Rose NR: Genetic complexity of autoimmune myocarditis. Autoimmun Rev 2008, 7:168-73.

26. Guler ML, Ligons DL, Wang Y, Bianco M, Broman KW, Rose NR: Two autoimmune diabetes loci influencing $T$ cell apoptosis control susceptibility to experimental autoimmune myocarditis. J Immunol 2005, I 74:2 I67-73.

27. Fairweather D, Frisancho-Kiss S, Rose NR: Sex differences in autoimmune disease from a pathological perspective. Am J Pathol 2008, I 73:600-9.

28. Frisancho-Kiss S, Nyland JF, Davis SE, Frisancho JA, Barrett MA, Rose NR, Fairweather D: Sex differences in coxsackievirus B3induced myocarditis: IL-I2Rbetal signaling and IFN-gamma 
increase inflammation in males independent from STAT4. Brain Res 2006, I I 26: 139-47.

29. Frisancho-Kiss S, Coronado MJ, Frisancho JA, Lau VM, Rose NR, Klein SL, Fairweather D: Gonadectomy of male BALB/c mice increases Tim-3(+) alternatively activated M2 macrophages, Tim-3(+) T cells, Th2 cells and Treg in the heart during acute coxsackievirus-induced myocarditis. Brain Behav Immun 2009, 23:649-57.

30. Fairweather D, Cihakova D: Alternatively activated macrophages in infection and autoimmunity. J Autoimmun 2009, 33:222-30.

31. Rose NR: Critical cytokine pathways to cardiac inflammation. J Interferon Cytokine Res 201 I, 3 I:705-10.

32. Fairweather D, Frisancho-Kiss $S$, Rose NR: Viruses as adjuvants for autoimmunity: evidence from Coxsackievirus-induced myocarditis. Rev Med Virol 2005, I5:17-27.

33. Guerder S, Joncker N, Mahiddine K, Serre L: Dendritic cells in tolerance and autoimmune diabetes. Curr Opin Immunol 2013, 25:670-5.

34. Afanasyeva M, Wang Y, Kaya Z, Park S, Zilliox MJ, Schofield BH, Hill SL, Rose NR: Experimental autoimmune myocarditis in A/J mice is an interleukin-4-dependent disease with a Th2 phenotype. Am J Pathol 200I, I59:193-203.

35. Baldeviano GC, Barin JG, Talor MV, Srinivasan S, Bedja D, Zheng D, Gabrielson K, Iwakura Y, Rose NR, Cihakova D: Interleukin-I7A is dispensable for myocarditis but essential for the progression to dilated cardiomyopathy. Circ Res 2010, 106:1646-55.
36. Barin JG, Baldeviano GC, Talor MV, Wu L, Ong S, Fairweather D, Bedja D, Stickel NR, Fontes JA, Cardamone AB, Zheng D, Gabrielson KL, Rose NR, Ciháková D: Fatal eosinophilic myocarditis develops in the absence of IFN- $\gamma$ and IL-I 7A. J Immunol 2013, 191:4038-47.

37. Fujinami RS, Herrath MG von, Christen U, Whitton JL: Molecular mimicry, bystander activation, or viral persistence: infections and autoimmune disease. Clin Microbiol Rev 2006, 19:80-94.

38. Hwang S, Song K, Lesourne R, Lee J, Pinkhasov J, Li L, El-Khoury D, Love PE: Reduced TCR signaling potential impairs negative selection but does not result in autoimmune disease. J Exp Med 20I2, 209: 178I-95.

\section{FlOOOPrime}

RECOMMENDED

39. Dighiero G, Rose NR: Critical self-epitopes are key to the understanding of self-tolerance and autoimmunity. Immunol Today 1999, 20:423-8.

40. Hamilton SR, Crome SQ, Ohashi PS: From Tolerance and autoimmunity - T cells. In The Autoimmune Diseases. 5th edition. Edited by Rose NR, Mackay, IR. San Diego: Elsevier; 2014:87-99.

41. Sakaguchi S, Rose NR: From Immune Mechanisms in Autoimmune Disease of Endocrine Organs. In Diagnosis and Pathology of Endocrine Diseases. Edited by Mendelsohn G. Philadelphia: Lippincott Williams and Wilkins; 1988:619-40.

42. Rose NR, Mackay I: From Autoimmune Disease: The Consequence of Disturbed Homeostasis. In The Autoimmune Diseases. 5th edition. San Diego: Elsevier; 20I4:3-9. 